\title{
Circulation de savoirs et espace d'intéressement
}

Types de savoirs dans un dispositif d'accompagnement

Circulation of knowledge and collaborative space: types of knowledge in a supportive plan of action

Circulación de saberes y espacio de implicación: tipos de saberes en un dispositivo de acompañamiento

Austausch von Kenntnissen und Raum des Teilhabens: Kenntnistypen bei Begleitungsmaßnahmen

\section{Patricia Tavignot}

\section{CpenEdition}

\section{Journals}

Édition électronique

URL : http://journals.openedition.org/rechercheformation/716

DOI : 10.4000/rechercheformation.716

ISSN : 1968-3936

Éditeur

ENS Éditions

\section{Édition imprimée}

Date de publication : 1 mai 2008

Pagination : 43-56

ISBN : 978-2-7342-1130-3

ISSN : 0988-1824

\section{Référence électronique}

Patricia Tavignot, «Circulation de savoirs et espace d'intéressement », Recherche et formation [En ligne], 58| 2008, mis en ligne le 01 mai 2012, consulté le 05 mai 2019. URL : http:// journals.openedition.org/rechercheformation/716 ; DOI : 10.4000/rechercheformation.716 


\section{CIRCULATION DE SAVOIRS ET ESPACE D'INTÉRESSEMENT Types de savoirs dans un dispositif d'accompagnement}

\section{Patricia TAVIGNOT*}

Résumé L'enjeu de cet article est d'analyser les circulations de savoirs dans un dispositif d'accompagnement "Recherche École primaire, académie de Roven ". Une circulation de savoirs au sein de l'« Équipe académique de Recherche » concerne l'accompagnement d'équipe d'école en démarche de recherche. De la circulation de savoirs entre chaque " binôme-équipe d'école " sont dégagés les types de savoirs. Enfin, nous dégageons que ces circulations de savoirs ont été dynamisées par le fait que ce dispositif s'interprète comme un espace d'intéressement.

Mots clés: enseignants, pratiques, circulation, savoirs.

Lors de l'opération « Recherche École primaire, académie de Rouen » (1999-2002), le dispositif d'accompagnement est défini comme un processus où l'accompagnateur et l'accompagné ne doivent pas se mettre à la place de l'autre. Cette opération est envisagée comme une totalité virtuelle comprenant l'« Équipe académique de Recherche » et tous les " binômes-équipe d'écoles".

La circulation de savoirs est portée par des acteurs issus de mondes différents qui impulsent la co-construction de nouveaux savoirs ou reproblématisent les savoirs présents. Dans ce dispositif d'accompagnement, nous repérons deux circulations de savoirs. La première, au sein de l'« Équipe académique de Recherche », concerne la notion d'accompagnement. La seconde, dans chaque " binôme-équipe d'école ", est dépendante du thème de recherche.

Après les présentations de ces deux circulations de savoirs, nous montrons qu'on peut comprendre la dynamique de ce dispositif si nous l'interprétons comme un espace $d^{\prime}$ intéressement.

* - Patricia Tavignot, IUFM de l'académie de Roven (CIVIIC). 


\section{CIRCULATION DE SAVOIRS DE L'« ÉQUIPE ACADÉMIQUE DE RECHERCHE ॥}

L'« Équipe académique de Recherche 》 s'est composée de trente-six personnes ayant différents statuts institutionnels: des inspecteurs de l'Éducation nationale, des conseillers pédagogiques de circonscription, des directrices d'école (ZEP, école d'application), des instituteurs maîtres-formateurs, des maîtres de conférences en Sciences de l'éducation et des formateurs IUFM. Chaque binôme d'accompagnement d'équipe d'école, constitué d'un chercheur et d'un praticien, a pu agir sans injonction, sans prescription et sans positionnement de pouvoir par rapport aux équipes d'école.

Pour I'« Équipe académique de Recherche », la ressource documentaire comprend vingt-cinq comptes rendus de réunions (CREAR) ainsi que vingt et un écrits sur la pratique de l'accompagnement (REDAC) de membres de cette équipe. De plus, sont pris en compte les quatre numéros du Journal de la Recherche (JR) et les actes des deux Forums des écoles (FE) avec les comptes rendus des ateliers thématiques (juin 2001, juin 2002).

La circulation de savoirs de l'« Équipe académique de Recherche », concerne l'accompagnement d'équipes d'école en démarche de recherche en distinguant relation d'accompagnement et posture d'accompagnement.

\section{La relation d'accompagnement}

Dans les comptes rendus, certains membres constatent l'importance de la relation d'accompagnement: comment respecter l'humain et répondre à ses attentes? Selon quelles modalités organiser cette relation? Ces constats se déclinent ainsi:

Une implication forte et familière:

"Il faut une plus grande implication de notre part pour créer un lien plus "intime" avec elles. " (plénière du 15 mars 2000)

Une implication distanciée où l'équipe d'école doit être demandeuse:

"Il y en a, plus formées à la méthodologie de la recherche qui "bidouillent", en revanche, elles contactent leur binôme et le perçoivent comme un soutien méthodologique pour la mise à disposition d'outils ou sur l'évaluation des dispositifs mis en place. " (plénière du 15 mars 2000)

Une incontournable confiance réciproque:

"Une confiance doit absolument s'instaurer entre l'équipe et le binôme, et cette confiance passe, à mon avis, par une participation minimum à la phase de mise en œuvre que ce soit dans la construction d'outils (grille d'observation,...) ou dans les expérimentations avec les élèves. 》 (REDAC de juin 2002)

La faisabilité de cette relation d'accompagnement dépend de la familiarité - distanciation avec le terrain. Elle se définirait comme une relation de proximité non 
fusionnelle. N'oublions pas que les rencontres " binôme-équipe d'école 》 se sont déroulées dans l'école hors des heures d'ouverture aux élèves. Ce choix a permis à certains formateurs de l'IUFM de mieux comprendre ce qu'est une école, ses besoins, son environnement et ses contraintes: "Pour moi, [il y a] un affinement de ma connaissance du terrain et des freins ou facilitations liés à la place des institutionnels (IEN, CPC,...) [et] l'identification de freins par rapport à l'enseignement des sciences ». (REDAC de juin 2002)

\section{La posture d'accompagnement}

En parallèle, certains comptes rendus témoignent de difficultés à se situer dans une posture d'accompagnement. Elles peuvent s'exprimer de la sorte:

Être dans une écoute synthétique et renvoyer les connaissances attendues:

"L'accompagnement nécessite beaucoup d'écoute mais trier dans ce flot de paroles, me pose un gros problème de méthodologie [...]. Il y a beaucoup de demandes par rapport aux techniques d'entretien, aux questionnaires à faire pour les enfants, les aides-éducateurs, je suis très embêtée par rapport à tout cela, c'est pourquoi j'ai fait cette demande au secrétariat... ". (plénière du 15 mars 2000)

\section{Un nécessaire engagement:}

"C'est lorsque nous sommes impliqués dans la démarche de l'équipe, au niveau du recueil des données que ce soit pour aider ou effectuer des entretiens, des observations, que la recherche avance. " (plénière du 14 mars 2001)

\section{Le rapport au temps modifié:}

"Il faut du temps pour travailler sur les réunions d'écoles pour analyser ce qui se dit, ce qui se fait "; "C'est difficile de gérer le rapport au temps, ne pas speeder, ne pas laisser courir... Quel est le point d'équilibre? C'est un fil tendu en permanence! ». (plénière du 19 janvier 2000)

Agir sans interférer l'activité de l'équipe d'école:

"Ill y a une complexité d'être à la fois spectateur et acteur. » (plénière du 15 mars 2000)

Les membres des binômes doivent accepter cette posture particulière, nouvelle pour certains d'entre eux:

"La posture est complexe car parfois on est tenté d'apporter un conseil pédagogique mais, faut-il conseiller quand on voit certaines choses... alors que c'est un accompagnement recherche? » (plénière du 6 décembre 2000);

"[la] place du conseiller pédagogique de circonscription [est] difficile à trouver. Le regard de part et d'autre n'est pas le même (école/binôme). Il est important que l'accompagnement se fasse hors [du] territoire de [la] circonscription ». (REDAC de juin 2002) 
Ce souci de positionnement face aux équipes d'écoles dans les premiers temps de l'accompagnement a été éprouvé par les membres conseillers pédagogiques: être proche tout en se distanciant du milieu d'enseignement premier degré.

De plus, le rapport au savoir du binôme est « chahuté » puisque l'équipe d'école est détentrice du contenu et le binôme n'y interfère qu'à sa demande. Le binôme doit accepter que l'équipe d'école ait plus de connaissances sur certains aspects en relation avec l'objet d'investissement. Le binôme est une aide pour réorganiser les savoirs.

La posture d'accompagnement ne se confond pas avec la posture de formation (plan de formation, élaboration de séquences à partir des attentes des stagiaires supposées par les formateurs, analyse de pratiques professionnelles). Les formateurs sont définis comme des passeurs de sens et de savoirs issus de la recherche en éducation ou de l'activité d'enseignement (Tavignot, 2004). Pour le formateur, membre d'un binôme, il y a dynamisation de la posture d'accompagnement par cette posture de formation et réciproquement. Les postures " $s^{\prime}$ imbriquent, se stimulent et $s^{\prime}$ enrichissent mutuellement », comme le souligne Lemoine (2005, p. 57). De plus, certains formateurs ont la recherche comme activité première. Dès lors pour eux, les positions du chercheur, du formateur et de l'accompagnateur sont enchevêtrées, comme le signale Canter Kohn (2001), il importe de bien déterminer le positionnement approprié selon la nature de l'activité conduite.

Donc, au fil du temps, cette circulation de savoirs quant à l'accompagnement s'effectue par une réflexion collective à partir des expériences individuelles. Cette pratique réflexive a permis, comme le souligne Cifali (2004, p. 82), " de rendre la parole du professionnel authentique lorsqu'il s'adresse à l'autre de la relation ». Cette appropriation, par les accompagnateurs, de savoirs pratiques et praxiques d'accompagnement facilite la poursuite de l'opération.

Examinons, maintenant, les circulations de savoirs des «binômes-équipe d'écoles».

\section{CIRCULATIONS DE SAVOIRS DANS LES « BINÔMES-ÉQUIPES D'ÉCOLE ॥}

Les " binômes-équipes d'école 》 sont des espaces restreints associant un binôme $d^{\prime}$ accompagnateurs et une équipe d'école, où l'on doit trouver des mots pour dire sa pratique à des tiers extérieurs, qui ont des statuts différents: un/des collègues et/ou le binôme d'accompagnement. Les équipes d'écoles peuvent être considérées comme des groupes professionnels questionnant directement l'activité réalisée, ce qui modifie la relation au travail: "L'idéal d'un praticien réfléchi a relayé les normes habituelles du travail prescrit. L'approche par l'action a permis un autre regard sur les gestes quotidiens. Une démarche clinique s'est confirmée, avec reconnaissance de la 
subjectivité, place faite à l'altérité, importance donnée au détail et au quotidien. 》 (Cifali, 2004, p. 69)

Pour les " binômes-équipes d'école ", nous disposons des dossiers "Équipe d'école ». Chaque dossier comporte une fiche signalétique de l'école (FSA) complétée par elle au cours de la dernière période de la recherche, les comptes rendus des visites (CRV) effectués par le binôme et les comptes rendus de stages (CRS) rendus par les formateurs. Dans certains dossiers ont été archivés les articles rédigés par l'équipe d'école et parus dans le Journal de la recherche, certains courriers lui étant adressés ou des notes du binôme. En vérifiant les quarante-quatre dossiers "Équipe d'école ", nous constatons que la fiche signalétique n'est pas vraiment renseignée. Treize dossiers d'école ont été tirés au hasard parmi les trente et un exploitables.

\begin{tabular}{|c|l|}
\hline Écoles & \multicolumn{1}{|c|}{ Thèmes des dossiers travaillés } \\
\hline 1 & Évaluation de l'autonomie des élèves. \\
\hline 2 & Autrement à la conquête du langage (pouvoir faire pour pouvoir dire). \\
\hline 3 & Le passage du statut d'enfant à celui d'élève. \\
\hline 4 & Le langage: développement du vocabulaire. \\
\hline 5 & $\begin{array}{l}\text { Situations et moyens mis en œuvre pour aider l'enfant à améliorer ses } \\
\text { compétences langagières. }\end{array}$ \\
\hline 6 & Aménagement du temps de l'enfant pour lutter contre l'échec scolaire. \\
\hline 7 & Apprentissage et remédiation. \\
\hline 8 & Améliorer le langage chez l'enfant. \\
\hline 9 & Amélioration du langage oral. \\
\hline 10 & Le langage oral. \\
\hline 11 & $\begin{array}{l}\text { Améliorer les performances et le développement de l'enfant en } \\
\text { aménageant le temps à l'école. }\end{array}$ \\
\hline 12 & Construire la citoyenneté à l'école. \\
\hline 13 & La communication orale. \\
\hline
\end{tabular}

Pour identifier les types de savoirs dans chaque circulation, nous retenons la distinction des cinq savoirs, développée par Van Der Maren (1996, p. 43 sq.):

- le savoir scientifique qui " a une prétention universelle»;

- le savoir appliqué qui est « une opérationnalisation du savoir scientifique »;

- "Le savoir stratégique, ou savoir pour l'action, [qui] se situe à l'enchevêtrement du savoir appliqué et de la praxis. [...] Le savoir stratégique est une sorte de savoir appliqué utilisable dans l'action, parce que ses règles sont plus immédiates, 
plus souples, plus concrètes, utilisant des signaux perceptibles plutôt que des instruments de mesure et des calculs ";

- le savoir praxique, issu de la praxis, qui « implique au préalable une réflexion sur la pratique [...] prépare à la pratique, elle permettra d'y accéder sans trop de surprises et sans trop d'erreurs ";

- le savoir pratique qui est « singulier, localisé, contextualisé, et la réalité dont il parle inclut l'homme avec son système de valeurs ».

\section{Des savoirs scientifiques peu nommés}

Ces savoirs identifiés comme scientifiques concernent les champs de la didactique/pédagogie et de la psychologie pour re/donner du sens à l'agir professionnel et proposer des repères conceptuels pour concevoir des situations d'enseignementapprentissage.

Pour le champ " didactique/pédagogie ", se repèrent des concepts de la didactique comme triangle didactique, statut de l'erreur et notion d'obstacle (école 1), puis des concepts au carrefour de la pédagogie et de la didactique : évaluation (école 5), obstacles à l'apprentissage du langage (école 10) et des concepts de la pédagogie: décloisonnement, travail entre cycles (écoles 2 et 8), concertation (écoles 2, 4 et 12). Pour le champ " psychologie ", certains concepts se situent en psychologie linguistique comme compréhension, explicitation et développement du langage lécoles 3 , 6, 810 et 13), d'autres plutôt en psychologie cognitive comme motivation et autonomie en termes de stratégies cognitives (écoles 1, 3, 4 et 12), enfin se dégagent des concepts comme chronobiologie et rythme scolaire (écoles 6 et 11) qui correspondent à une des entrées de la « Charte pour bâtir l'École du XXle siècle ".

L'absence de traces de savoirs scientifiques "labellisés » ne signifie pas que les équipes d'école y soient indifférentes. Après l'opération, certaines équipes ont été demandeuses de références bibliographiques.

\section{Des savoirs appliqués: reflets de mobilisation de savoirs scientifiques}

Il est possible d'inférer des éléments d'usage de savoirs scientifiques dans les savoirs appliqués. Deux savoirs appliqués se dégagent: un méthodologique et l'autre conceptuel.

Le savoir appliqué méthodologique permet le développement de méthodes d'investigation et d'observation de la recherche fondamentale : questionnaires, entretiens et grilles d'observation (sauf écoles 5, 6 et 8). L'outil se conçoit collectivement et la passation se réalise par chaque enseignant dans sa classe. 
L'autre savoir appliqué conceptuel concerne l'hétérogénéité pour une meilleure prise en compte des élèves:

- soit en renforçant l'implication des élèves: avec l'auto-évaluation (école 1), pour favoriser une motivation interne par le décloisonnement (école 4) ou en dynamisant le tutorat (écoles 7 et 11);

- soit pour une meilleure prise en compte des capacités intellectuelles des élèves: par l'explicitation des stratégies cognitives des élèves (écoles 1), avec la notion d'activité langagière (école 2), en se focalisant sur le repérage d'indicateurs de compréhension langagière (écoles 3), en abordant la notion de groupes de niveaux (école 9), par une formation à " la pédagogie du langage » (école 10) et en construisant le sens de la loi (écoles 11 et 12).

\section{Le travail d'équipe: un savoir stratégique spécifique}

Nous repérons une constante dans les traces relevées: la centration sur le travail d'équipe. La prégnance de son développement et de son maintien, comme l'apparition d'une nouvelle appréhension de cet engagement sont évoquées explicitement ou implicitement:

- évocation affirmée par l'usage des termes: travail en équipe/d'équipe lécoles 3 , $4,5,6,8,10$ et 11), cohésion/cohérence de l'équipe (écoles 9,10 et 12);

- évocation d'actions réalisées: construction de scénarios pédagogiques, d'activités didactiques (école 1), changements concertés des modalités de regroupement pour les ateliers de pratiques langagières (école 2), stratégie collective d'observation des comportements des élèves (école 3 ), élaboration d'outils d'évaluation ou d'évaluations communes (écoles $4,5,7$ et 10), co-construction de règles de vie et de contrats individuels de comportement (école 12) et harmonisation des pratiques (école 7).

\section{Mode de fonctionnement ou choix pédagogique comme savoir praxique}

Pour sept " binômes-équipes d'école ", nous repérons des traces d'une étape de réflexion quant au mode de fonctionnement ou au choix pédagogique en relation avec le thème de recherche.

Des modes de fonctionnement: modification de l'accueil des élèves (école 3), mutualisation des activités langagières (école 10), et mutualisation des pratiques (école 11). Des choix pédagogiques: création de groupe de besoin (école 1) ou de petits groupes de langage (école 5), élaboration d'activités langagières (école 2), et création de bilan d'auto-évaluation (école 12). 
L'explicitation des modes de fonctionnement ou des choix pédagogiques amène à certaines décisions de changement qui peuvent toucher tout ou partie de l'organisation de l'école ou les actes professionnels individuels.

\section{Des savoirs pratiques reproblématisés}

Les savoirs pratiques évoluent ou émergent lors des rencontres " binôme-équipe d'école » selon la temporalité de la démarche de recherche. Ces savoirs pratiques concernent l'organisation de l'école et/ou l'activité d'enseignement-apprentissage:

- l'organisation de l'école: un partage coopératif pour la conquête du langage (école 2), une gestion des emplois du temps pour dynamiser le passage du statut d'enfant à celui d'élève (école 3), le décloisonnement pour améliorer le langage chez l'enfant (école 8), le développement du partenariat avec les parents et l'aménagement de l'emploi du temps pour lutter contre l'échec scolaire (école 6) ou des conseils d'élèves et un contrat éducatif local avec une cellule d'écoute pour une construction de la citoyenneté à l'école (école 12);

- l'organisation de l'école associée à une approche collective de l'activité d'enseignement-apprentissage: le décloisonnement avec des ateliers d'écriture pour aider l'enfant à améliorer ses compétences langagières (école 5), le décloisonnement avec l'utilisation de cassettes pour l'amélioration du langage oral (école 9), un travail par cycle et une réflexion interniveaux pour l'apprentissage du langage oral (école 10), ou des échanges interclasses et intercycles selon les domaines de compétences quant aux matières à enseigner afin d'améliorer les performances et le développement de l'enfant (école 11);

- I'activité d'enseignement devant les élèves: une prise en compte effective des obstacles à l'apprentissage pour l'évaluation des élèves (école 1), une verbalisation des élèves plus systématique pour développer le vocabulaire (école 4) ou le jeu théâtral et la réalisation de vidéo pour renforcer la communication orale (école 13).

Donc, les savoirs scientifiques sont peu nommés mais semblent présents implicitement. Le sens de concepts est manifestement construit dans les équipes où un enseignant possédait des compétences universitaires en matière de recherche (écoles 1, 6 et 13). Des reflets de mobilisation de certains savoirs scientifiques dans des savoirs appliqués sont identifiés même si l'étiquette des concepts n'est pas systématiquement posée. L'implication dans la démarche de recherche des équipes d'école a permis aux enseignants de construire un savoir stratégique: le travail d'équipe avec une mise en œuvre effective. L'explicitation de modes de fonctionnement ou de choix pédagogiques est au centre du savoir praxique co-construit dans les " binômeséquipes d'école ". Dès lors, certains savoirs pratiques émergent ou d'autres sont en fait des reproblématisations de savoirs pratiques existants collectivement ou indivi- 
duellement. D'ailleurs, les actes du dernier forum des écoles (FE de juin 2002) se font l'écho de cet état quand les enseignants explicitent leur regard sur le dispositif recherche: "L'accompagnement a introduit une prise de recul sur les pratiques et une mise en forme des questionnements d'équipe. Les sollicitations ou les aides visant à cette reformulation ont conduit à expliciter davantage qu'à découvrir les problématiques de l'école. Le binôme, par sa simple présence ou ses interventions pratiques dans l'action (conduite d'entretien), est un soutien jugé indispensable. »

L'appropriation des savoirs scientifiques n'est pas « visible " dans les traces écrites des dossiers d'écoles, mais une forme d'opérationnalisation plus ou moins explicitée peut être dégagée. Prenons l'exemple de l'école 1 qui a travaillé sur l'autonomie des élèves. Les membres de l'équipe d'école en présence du binôme mettent en commun leurs connaissances d'expérience autour de l'autonomie (savoirs pratiques, praxiques), il y a élaboration d'un sens collectif sur l'autonomie puis mise en pratique en direction des élèves. Ensuite, il y a une prise de recul avec l'intervention des accompagnateurs; ce qui amène l'équipe à construire des savoirs stratégiques sur l'autonomie. Dans un dernier temps, une réflexion est engagée sur l'évaluation de l'autonomie des élèves et le concept d'autonomie (savoir scientifique).

Une dialectique cyclique semble se dégager entre circulation et construction de savoirs. Après la phase de négociation conceptuelle autour de l'objet thématique, les équipes semblent $d$ 'abord s'investir dans une phase de circulation de savoirs avec un double processus mutualisation/réflexion, celle-ci serait suivie d'une phase de coconstruction de sens sur certains savoirs repérés et faisant unanimité, puis d'autant d'enchaînements nécessaires de ces deux moments, avec des processus variés.

\section{LE DISPOSITIF D'ACCOMPAGNEMENT COMME ESPACE D'INTÉRESSEMENT}

La notion d'intéressement recouvre une interaction entre un intérêt individuel et une action collective, pour le mieux être de l'un et de l'autre. Elle a été reprise, en sociologie des sciences, par les chercheurs du Centre de sociologie de l'innovation de l'école des Mines, dans des travaux sur les conditions de réussite de l'innovation. Ils y ont précisé le modèle de l'intéressement: "Le modèle de l'intéressement met en scène tous les acteurs qui se saisissent de l'objet ou s'en détournent, et il souligne les points d'accrochage entre l'objet et les intérêts plus ou moins organisés qu'il suscite " (Akrich, Callon, Latour, 1991, p. 51). L'espace d'intéressement fonctionne de façon démocratique. "L'égalisation relative des "titres à parler", l'opportunité donnée à tous les porte-parole $d^{\prime}$ 'argumenter pour eux-mêmes et $d^{\prime}$ interroger les justifications 
des autres, transforment pour un temps les hiérarchies ordinaires et les conceptions qui les sous-tendent » (Callon, Lascoumes, Barthe, 2001, p. 58). De plus, I'espace $d^{\prime}$ 'intéressement unit des acteurs mus par certaines intentionnalités ou par certains intérêts propres ou communs d'où la présence d'une "zone d'intersubjectivité » (Tavignot, 2004).

La circulation de savoirs dans des espaces d'intéressement constitue un sujet porteur de développements potentiels dans une nouvelle orientation paradigmatique. Il s'agit d'un nouveau mode de circulation/diffusion des résultats de la recherche visant une plus grande appropriation par les praticiens. En même temps, il porte un rapprochement du monde de la recherche du terrain, des enseignants et de leurs savoirs pratiques, et donc une adéquation entre les champs de recherche et les domaines de préoccupation des enseignants.

Dans l'opération " Recherche École primaire », le dispositif d'accompagnement a joué un rôle structurant pour faire fonctionner la recherche comme un espace d'intéressement, qui a permis et dynamisé la circulation de savoirs entre les acteurs présents. Cette émergence de réseaux entre les enseignants, les formateurs et les chercheurs crée des conditions de dialogues, de coopérations et d'ouvertures réciproques entre ces acteurs.

Si nous cherchons à dégager les traits saillants du dispositif qui ont constitué ces conditions nécessaires de ce fonctionnement, nous pouvons relever:

- la volonté et la stabilité des acteurs (institutions comme individus);

- le soutien matériel et institutionnel;

- la fonction miroir de l'accompagnement.

Conditions qui s'avèrent nécessaires à une réelle circulation de savoirs au sein de l'«Équipe académique de Recherche » et dans les « binômes-équipes d'école ».

\section{Conditions au sein de l'« Équipe académique de Recherche"}

\section{Volonté et stabilité des acteurs}

Les membres volontaires de l'« Équipe académique de Recherche » s'impliquent pour des motivations variées et pas forcément au début avec des intérêts conceptuels. Signalons la présence d'un " noyau de permanents 》 (dix-neuf personnes) de I'« Équipe académique de Recherche » sur les trois années scolaires de la recherche. $\mathrm{Ce}$ « noyau de permanents » a favorisé la dynamique de cette équipe et a permis de tenir une réelle mémoire collective et non une juxtaposition de mémoires individuelles. 


\section{Soutien matériel et institutionnel}

L'opération a bénéficié du soutien du rectorat de l'académie de Rouen. Dès le début en 1999, M. le Recteur a engagé l'ensemble des services académiques pour favoriser la démarche du dispositif de recherche et d'accompagnement.

L'IUFM s'est investi dans la conduite de cette opération en mettant à sa disposition un demi-temps de secrétariat et les ressources matérielles nécessaires au fonctionnement. De plus, les déplacements des membres de l'équipe ont été pris en charge pour ceux du premier degré (CPC, IMF, PE) par l'inspection académique et pour les formateurs IUFM et les enseignants-chercheurs de l'université par l'IUFM.

Depuis le début de l'opération, l'INRP a mis à la disposition de l'équipe académique de recherche un mi-temps d'enseignant du premier degré. Pour renforcer le dispositif lors de la dernière année, I'INRP a octroyé un autre mi-temps.

\section{Fonction miroir de l'accompagnement}

L'accompagnement deviendrait un dispositif de collaboration qui respecterait les activités des professionnels: "[la démarche d'accompagnement] serait [une source d'enrichissement professionnel] si l'on pouvait mettre en place des moments d'observation en classe et de collaboration plus régulière avec les écoles. Je pense que c'est une mission importante des formateurs IUFM ». (REDAC - FG de juin 2002) L'accompagnement a eu des effets sur l'identité professionnelle de l'ensemble des membres de l'« Équipe académique de Recherche ». Martineau et Gauthier (2000) écrivent: "Parce que l'identité professionnelle est associée au rapport à autrui, sa modification passe souvent par la rencontre avec l'altérité » (p. 89). Une formatrice IUFM le déclare: "Pour ma part, ce fut une expérience fructueuse qui a permis d'enrichir ma compétence professionnelle grâce aux liens qui ont pu se constituer avec le terrain. » (REDAC de juin 2002)

L'« Équipe académique de Recherche » a favorisé la transformation identitaire des individus en présence par la circulation du savoir quant à l'accompagnement. Cette transformation est accentuée des pratiques personnelles de chacun.

\section{Conditions dans les " binômes-équipes d'école "}

\section{Volonté et stabilité des acteurs}

Les écoles participant à l'opération ont été volontaires. Il est possible de distinguer des degrés de volontariat: le volontariat spontané et le volontariat sollicité. Dès que l'équipe d'école est changée (départ à la retraite ou mutation), elle sort du dispositif. Lorsque le binôme d'accompagnement est modifié, il y a déstabilisation du processus d'accompagnement. 


\section{Soutien matériel et institutionnel}

L'implication de l'institution par rapport aux moyens alloués pour le fonctionnement de l'espace d'intéressement est primordiale, notamment du temps libéré qui favorise la présence effective de chacun aux différentes rencontres. Comme nous l'avons constaté avec ce dispositif d'accompagnement d'équipe d'école en démarche de recherche, de nombreux moments de rencontres s'imbriquent: rencontres entre membres de l'équipe d'école, rencontres avec le binôme d'accompagnement (prévues ou sollicitées) et stages de formation demandés.

\section{Fonction miroir de l'accompagnement}

Au gré des interactions entre les acteurs de l'équipe d'école et le binôme d'accompagnement, une lecture théorique de l'activité réalisée se conçoit. L'équipe d'école a élaboré son objet d'investissement, pour le délimiter elle est épaulée par le binôme. On se situe plus dans une problématisation de l'activité réalisée avec production de savoirs professionnels: "Le regard extérieur apporté par les membres du binôme $d^{\prime}$ accompagnement est absolument nécessaire même si la recherche doit continuer à appartenir à l'équipe d'école de bout en bout. " (Forum des écoles, $n^{\circ} 2$ 2) Les acteurs impliqués s'accordent une confiance réciproque. Chacun s'engage à respecter conceptuellement les interprétations d'autrui. En étant dans une position de compréhension mutuelle, les acteurs investissent un travail sur le langage notamment sur le sens des termes utilisés. Des écrits sont rédigésés pour assurer la mémoire collective de l'espace d'intéressement. Un écrit final est à diffuser aux institutionnels, aux pairs et aux chercheurs.

54 Effectivement, les membres des binômes ont des statuts différents et n'ont aucune relation de hiérarchie institutionnelle ou de formation avec les équipes d'école. Cette condition évite une proximité trop paralysante pour tous et conduit les membres du binôme à se distancier des habitus de leur propre activité professionnelle.

Donc, ces conditions permettent aux acteurs de l'espace de dépasser le processus de négociation pour stabiliser les intérêts de chacun. Les relations qui s'établissent alors, renforcent l'entrée dans les processus de communication de savoirs, de mobilisation de savoirs et co-construction d'autres savoirs d'où circulation de savoirs. Les motivations $d^{\prime}$ intéressement régulent les interactions exploratrices de sens. Elles sont à dynamiser, à entretenir. Elles peuvent l'être par l'individu lui-même, par le collectif ou par le contexte. 


\section{En conclusion}

Dans cette recherche, nous avons vu fonctionner deux dispositifs, I'un concernant des praticiens en démarche de recherche, l'autre des accompagnateurs de ce premier dispositif. C'est dans l'articulation de ces deux dispositifs que s'est construit l'espace d'intéressement étudié. Il a permis aux acteurs de " se distancier 》 de leur pratique professionnelle. Les écrits des acteurs montrent qu'ils ont ressenti ce changement comme une évolution constructive et efficace, car elle prenait en compte leur " espace-temps » professionnel et s'inscrivait bien dans leur communauté de travail. Ils pouvaient donc continuer d'inventer des solutions locales aux problèmes qu'ils rencontraient dans leur pratique, sans pour autant $s^{\prime} y$ absorber. Comme le dit Wenger $(2005$, p. 55) : « La pratique doit être interprétée comme un processus d'apprentissage » mobilisateur. Dans les pratiques professionnelles, on peut lire l'histoire capitalisée de ce processus, pour peu qu'un dispositif permette de le suivre comme cela a, ici, été fait.

\section{BIBLIOGRAPHIE}

AKRICH M., CALLON M., LATOUR B. (1991). « L'art de l'intéressement », in D. Vinck [coord.], Gestion de la recherche. Nouveaux problèmes, nouveaux outils, Bruxelles: De Boeck Université, p. 27-52.

CALLON M., LASCOUME P., BARTHE Y. (2001). Agir dans un monde incertain: essai sur la démocratie technique, Paris: éd. du Seuil.

CANTER KOHN R. (2001). " Les positions enchevêtrées du praticien qui devient chercheur 》, in M.-P. Mackiewicz [coord.], Patricien et chercheur. Parcours dans le champ social, Paris: L'Harmattan, p. 15-38.

CIFALI M. (2004). "Variations autour d'un dispositif d'enseignement: écrire et raconter des histoires ", in C. Lessard, M. Altet, L. Paquay, P. Perrenoud [éd.], Entre sens commun et sciences humaines. Quels savoirs enseigner? Bruxelles: De Boeck Université, p. 69-91.

LEMOINE M. (2005). « D'une démarche professionnelle à une démarche scientifique : filiation puis autonomie de la recherche sur un terrain familier », Les sciences de l'éducation pour l'ère nouvelle, vol. 38, $n^{\circ} 1$, p. 43-59.

MARTINEAU S., GAUTHIER C. (2000). « La place des savoirs dans la construction de l'identité professionnelle collective des enseignants ou le paradoxe de la qualification contre la compétence », in C. Gohier, C. Alin [dir.], Enseignant-formateur: la construction de l'identité professionnelle. Recherche et Formation, Paris: L'Harmattan, p. 85-110.

TAVIGNOT P. (2004). « Circulation des savoirs issus de la recherche et de la pratique: I'émergence d'un espace d'intéressement en formation initiale transversale d'enseignants du second degré ", 5e Congrès de l'AECSE, Paris: CNAM, Site AECSE [9 p.]. 
Circulation de savoirs et espace d'intéressement

TAVIGNOT P. (2005). « La professionnalisation : un paradoxe ou un consensus au service de l'expérience? », in R. Wittorski [coord.], Formation, travail et professionnalisation, coordination, Paris: L'Harmattan, p. 173-194.

VAN DER MAREN J.-M. (1996). Méthodes de recherche pour l'éducation, Bruxelles: De Boeck Université.

WENGER E. (2005). La théorie des communautés de pratique. Apprentissage, sens et identité, Laval: Les Presses de l'université Laval [Québec]. 